\title{
Rare Diseases in India: Current Knowledge and New Possibilities
}

\author{
SUDHA BHATTACHARYA ${ }^{1, *}$, VISHWA MOHAN KATOCH ${ }^{2}$, PARTHA P MAJUMDER $^{3}$ and ALOK \\ BHATTACHARYA ${ }^{4}$ \\ ${ }^{1}$ School of Environmental Sciences, Jawaharlal Nehru University, New Delhi, India \\ ${ }^{2}$ NASI-ICMR Chair on Public Health Research at RUHS, Jaipur, Rajasthan, India \\ ${ }^{3}$ National Institute of Biomedical Genomics, Kalyani, West Bengal, India \\ ${ }^{4}$ School of Life Sciences, Jawaharlal Nehru University, New Delhi, India
}

(Received on 17 May 2016; Accepted on 25 May 2016)

Notwithstanding that the ultimate goal of science is pure pursuit of the unknown, most scientists do find greater fulfilment, when through their knowledge they can reach out and touch lives of fellow humans for the better. In that spirit, Indian National Science Academy, through its Science and Society programme, sponsored a two-day workshop entitled "To Develop a Scientific Program for Research on Rare Diseases" on April 22-23, 2016 at INSA premises. The workshop was co-sponsored by Jawaharlal Nehru University, New Delhi and World without GNE-Myopathy (India). There were 22 presentations during the workshop. Besides these, one panel discussion on "Current status and future directions" was also organized along with a special lecture on policy issues. The Workshop ended with a meeting of all stakeholders, which included patients, clinicians, scientists and drug companies.

Rare diseases (as they are called) rarely figure on the scientific agenda of our country. This workshop was perhaps the first attempt in India to bring together experts and stakeholders in diverse areas related to rare diseases to comprehensively deliberate on issues beginning with the very definition of what constitutes a "Rare disease", to awareness of rare diseases among clinicians and lay public, possibilities of research in diagnostics and therapy using state-ofthe-art technologies, relief and rehabilitation of patients by existing clinical practices, policy framework for boosting and incentivising $R \& D$ efforts in treatment options, and introduction of suitable legislation to ensure involvement of the State in fulfilling the special needs of rare diseases. Prof. P. N. Tandon (past President, INSA), in his inaugural address, observed that the 'rareness' of a disease is a dynamic concept. Infectious diseases that were common in the past may be rare, or even eradicated today. Conversely, diseases not known to occur in India in the past have begun to be recorded once clinicians became aware of their special diagnostic features.

\section{Rare Disease Definition}

While it is obvious that, 'Rare' is a relative numeric, it is useful for many purposes to give a certain definition or cut-off value at which to place a disease in the rare category. Recognizing the difficulty of stating such a number for a relative entity, Dr. Katoch informed that as many as 296 definitions exist the world over for rare diseases. A large number of speakers quoted the generally accepted international figure that a disease may be considered "rare" if it affects less than 4-5 individuals per 10,000. India is yet to adopt an official definition in this context. Quoting from international sources several speakers informed that the number of different rare diseases in the world is estimated to range somewhere in between 6000 to 8000 . Though diseases termed as "rare" may be rare individually, together they affect $5-7 \%$ of populations in different countries, including India. This number is debatable as Dr. I. C. Verma (Sir Gangaram Hospital, New Delhi), a pioneer in human genetic disorders, was of the view that the

*Author for Correspondence: E-mail: sbjnu110@gmail.com 
number could be exaggerated. However, given the fact that $\sim 80 \%$ of known rare diseases are due to genetic mutations, and that different mutations in a single gene could lead to variable phenotypes, the number of rare genetic diseases is expected to be high. Whatever the number, India lags behind in reporting rare disease occurrence, and Dr. Katoch informed that so far only $\sim 450$ rare diseases have been recorded in India. These encompass a whole range of disorders, including primary immune deficiencies, blood disorders, metabolic disorders, muscular dystrophies and myopathies, neurological, skeletal, and mental disorders, and many more. With a thrust on increasing awareness for preventive and management purposes, he stressed on development of information/training modules for public, doctors/ specialists, practitioners, other health care providers and policy makers so that awareness is increased and programmes for their diagnosis and management are developed/strengthened. Prof. ParthaMajumder commented that amongst the rare diseases the very rare ones could be manifestation of one underlying gene, while the more common would be due to multiple possible genes. He informed that $\sim 300$ new disease genes are being identified every year through human exome or genome sequencing, getting us closer to defining the genes, or gene-combinations involved in particular phenotypes.

\section{Current Clinical Scenario in Rare Diseases: Indian Experience}

The meeting saw a congregation of leading clinical practitioners of rare genetic disorders in India who described their major findings and ongoing efforts. Dr. Madhulika Kabra (AIIMS, New Delhi) described the AIIMS experience in rare diseases and noted that over the years they are receiving more and more referrals, indicating a rise in the general awareness of rare genetic diseases. They have been focusing on selected diseases like cystic fibrosis (CF), Rett syndrome, megalencephalic leukoencephalopathy (MLC), deafness, disorders leading to intellectual disability and developmental delay. They use a combination of whole exome sequencing, clinical exome, and chromosomal microarray for definitive diagnosis, and have reported a number of novel mutations in Indian patients that were not known so far. Their work revealed a founder MLC mutation in the Aggarwal community, and highlighted the difficulty of firmly establishing genotype-phenotype correlation due to the rare occurrence of many mutations. CF was thought to be very rare in India, but genetic analysis has now shown that the disease is prevalent, but was undiagnosed earlier. The common mutation, DF508 in the Caucasians is found in India but at a lower frequency. There is a need to determine the common Indian mutations leading to $\mathrm{CF}$ and estimate the carrier frequency.

Dr. Shubha Phadke (SGPGI, Lucknow) narrated the hugely successful haemophilia (HA) program in UP funded by the State government. The incidence of HA in India is estimated to be 1 in 5,000. The disease can be treated by administration of blood clotting factors which are available as recombinant proteins, but the costs are prohibitive. In this context, it is heartening that, in 2010, the UP Govt. sanctioned generous funding to cover the cost of clotting factor procurement. An objective assessment of the outcome of treatment on the quality of life of HA patients is yet to be done.

Dr. Amita Aggarwal (SGPGI, Lucknow) described a rare presentation of auto-inflammatory syndrome with periodic fever and abdominal pain. Through a systematic analysis, they could determine the involvement of IL-1 pathway and could bring relief to the patient with suitable IL-1 inhibitors. Diagnosis was confirmed by mutation analysis.

Dr. Sumita Danda (CMC, Vellore) described the ongoing work in the Medical Genetics department set up in 2004. They see more than 3000 new cases of genetic disorders every year. Typically, they carry out karyotype analysis and FISH on patients with dysmorphology, developmental delay and other malformations. The disorders identified in their centre include Prader-Willi syndrome, MAP kinase-related RASopathies, neuromuscular and neurological disorders. Their centre reported the common occurrence of alkaptonuria, a rare genetic disorder in the gypsy population of Tamil Nadu and found a new splice site mutation, not identified before.

Dr. Shyamal Das (SSKM hospital, Kolkata) described his experience with patients of neurodegenerative diseases, particularly Huntington's and Parkinson's diseases. He apprised about current diagnostic methods and the need for effective therapies, especially for HD. 
Dr. Ratna D. Puri (Sir Gangaram Hospital) focuses on lysosomal storage disorders (LSDs) which collectively occur 1 in 5000 individuals. The phenotypes could result from loss of one of 50-odd enzymes of the lysosome, and the disease typically involves multiple organs. Their centre performs enzyme assays followed by exome sequence for confirmed diagnosis. Their studies showed the role of autophagy in disease pathology. Possible therapies include: pharmacologic chaperones to enhance correctly folded proteins, stem cell transplant at early stage of disease, and enzyme replacement therapy, which is available through recombinant enzymes, but is prohibitively expensive.

Dr. Debashish Choudhury (G. B. Pant Hospital, New Delhi) described rare CNS infections due to protozoa, particularly Naegleria, Acanthamoeba, Balamuthia and Sappinia. These are extremely rare and therefore, are generally missed with fatal consequences. His team has successfully treated many such patients. Naegleria fowleri enters through nasal mucosa and goes to the brain through the olfactory nerve. Out of 10 reported cases in India, only 2 survived. A common source of infection is through contaminated water in swimming pools and other water bodies. He suggested that simple warning sign at pools alarming swimmers to swim with nose above water could help reduce the incidence.

It has become clear from the discussion that whenever proper diagnostic methods have been put in place, more people get diagnosed and awareness about the disease also spreads.

\section{Technology Platforms for Diagnosis and Therapy: Ongoing Programmes in India}

The speed and progressively reducing cost of DNA sequencing makes it a practical reality to diagnose rare genetic diseases by direct sequencing. The promise of technologies involving stem cells and mutation correction by gene editing continue to hold hope for rare disease patients. The meeting brought together scientists from both the private and public sectors to talk about their goals and achievements.

Dr. Ramesh Hariharan (Strand Life Sciences, Bengaluru) highlighted important issues in sequence analysis for disease diagnosis. He emphasized that association of mutation with disease phenotype is not always straightforward because mutations are seen in normal people as well. Mis-sense mutations in evolutionarily conserved regions are more likely to be disease-associated. Single nucleotide change in an intron could be important. One such mutation was associated with a thalassemia condition, as it resulted in change of splice junction. A synonymous change in the exon, occurring at exon-intron boundary, led to splice variation in a Fanconi's anemia case. A large deletion in one allele may be missed since the sequence is provided by the other allele. Pseudogenes also pose a challenge as seen in diagnosis of colour blindness.

Dr. Ravi Gupta (MedGenome Labs, Kochi) described his company's mission to make a genome sequence database, called South Asian Genome Variation Database, which should represent the great genotypic diversity of our population. They need to include individuals of each ethnicity for a more complete picture. So far they have not done sequencing of their own but have accessed data from $215 \mathrm{~S}$. Asian individuals from public databases. They have done rare variant annotation and find a lot of diversity. According to GO analysis, they found variations enriched in sensory perception genes.

Dr. Vinod Scaria (IGIB, New Delhi), along with Dr. Sridhar Sivasubbu of the same Institute, set up a CSIR-funded project, called Genomics for Understanding Rare Diseases India Alliance Network (GUaRDIAN), with the aim to bring together clinicians and basic science researchers to understand novel genetic variations to achieve translational applications. Their approaches include: NGS sequencing and computational analysis on the one hand, and disease modelling using zebrafish system on the other. They are also engaged in patient support and education, and running training workshops for exome sequence analysis.

Dr. R. V. Shaji (Centre for stem cell research, CMC, Vellore) is setting up stem cell as a possible therapy for Fanconi's anemia (FA) patients. FA affects the DNA repair pathway, and 18 different genes are known to be involved. It is a bone marrow failure disease with cells showing cell cycle arrest and chromosome breaks. They have made iPSCs (induced pluripotent stem cells) which can be stored. Mutant iPSC cell lines can be used as efficient disease models in vitro. They plan to differentiate iPSCs into 
haematopoietic stem cells for possible therapeutics.

Dr. Aroon Sastry (The Dystrophy Annihilation Research Trust, Bangalore) talked about the initiatives by DART - a Trust started by a Duchene muscular dystrophy (DMD) patient's family, to find cure for DMD. As is well known, dystrophin is a very long gene with 79 exons. He apprised of the various clinical trials going on internationally for a variety of potential treatments, including utrophin-upregulating molecules, myostatin which can increase muscle mass, and gene therapy. Since the gene is very large, there are efforts to correct parts of the gene. For example, skipping of defective exons could lead to shorter but functional dystrophin. They have initiated work in skipping of exon 51 by using antisense oligonucleotides, and will extend this approach to other exons too.

Dr. G. N. Singh (Drug Controller General, India) stated that a policy for accelerated clearance of orphan drugs and fast-track approval is not in place because Govt needs clear-cut recommendations relating to what is definition of rare disease, mechanism for fast track approval (e.g. waive phase 1 for orphan drug trial), genetic differences in Indian populations which may warrant special studies instead of using known drugs from western studies. Participation of Indian patients in international trials need to be justified. He called upon experts to suggest specific changes in Drugs and Cosmetic act, which will meet the current needs of development of diagnostics/therapeutics for rare diseases. He promised that the recommendations of this workshop/expert groups will be considered for implementation.

\section{Epidemiology of Different Rare Diseases}

There is no clear idea about the number of patients afflicted with different rare diseases in India. There have been no efforts to carry out a systematic epidemiological study on rare diseases. Given the complexity of different diseases and difficulty in diagnosis, it is probably a herculean task to attempt a pan India study. However, some groups are carrying out limited studies on a few diseases in their locality/ state and have collected valuable data through their determined efforts. Dr. B R Lakshmi (Molecular Diagnostic, Counselling Care and Research Centre, Coimbatore) has focused on DMD (Duchene muscular dystrophy) and SMD (spinal muscular dystrophy) and through innovative approach has surveyed over 16 lakh households in a number of districts in Tamil Nadu for occurrence of these two diseases. She has so far diagnosed 4,500 confirmed cases of DMD, pointing to a high prevalence of the disease in Tamil Nadu. Dr. P Patra (Pt JNM Government Medical College, Raipur) studies sickle cell disease and has carried out systematic epidemiological studies in a few districts of Chhattisgarh, where this disease is prevalent in the tribal population.

\section{Understanding the Molecular Basis of Disease Pathology: New Initiatives}

For many rare diseases, the underlying biological mechanisms that lead to a specific pathology are not known though the causal mutations have been identified. We need to carry out research in understanding disease pathology since the results would not only help in developing suitable therapeutics, but also enrich scientific knowledge.

Dr. Chandan Goswami (NISER, Bhubaneshwar) is focusing to study Olmsted syndrome- a rare keratin disorder with unclear genetic basis. Mutations in TRPV3 (Transient receptor potential vanilloid-3) gene have been identified as a cause of OS, although other genes may also be involved. Rare, but naturally occurring mutations in TRPV channels are known to induce several critical pathophysiological disorders due to altered channel function, regulation and/or distribution. They have made TRPV3 mutant cell lines as a model system and show lysosomal defects and problems of calcium buffering in mutants. The 1000 genomes data shows that this channel sequence is highly divergent in the human population.

Dr. Ranjana Arya (Jawaharlal Nehru University, New Delhi) presented her recent data on GNE myopathy, a rare disorder due to mutations in the glucosamine (UDP-N-acetyl)-2-epimerase/Nacetylmannosamine kinase (GNE) gene. The protein encoded by this gene is a bi-functional enzyme involved in sialic acid biosynthetic pathway. They have generated cell lines with mutations in GNE gene and made the significant observation that hyposialylation may not be the only cause of pathology, and the GNE protein may be involved in other pathways as well. They find increased apoptosis in the mutant cells with abnormal mitochondrial morphology and ER stress. 
Apart from cell lines, they are also studying patient muscle biopsies obtained from Dr. A. Nalini (NIMHANS, Bangalore). She further showed that modified sialic acid has potential to be a good therapeutic agent.

Dr. Upendra Nongthompa (I.I.Sc., Bengaluru) is also interested in myopathies and is developing both Drosophila and zebra fish as animal models to study various myopathies. He presented his data with protein aggregate myopathies (PAM), in collaboration with Dr. Nalini and Dr. Gayathri (NIMHANS, Bengaluru), using a Drosophila model. He showed interesting application of Raman spectroscopy to differentiate muscle fibres from healthy and myopathic flies. Using this method, they could differentiate between nemaline myopathy and cardiomyopathy, and it also worked for a human muscle sample. This holds promise as a diagnostic tool, requiring a very small amount of tissue. They have made similar advances using laser Doppler vibrometry, and are also studying the efficacy of Ayurvedic formulations for myopathies.

Dr. Sridhar Sivasubbu (IGIB, New Delhi) described the zebra fish model system set up by them to study a whole range of human diseases. They could mimic the human disease phenotype in transgenic zebra fish. They are studying role of non-coding RNAs in cardiovascular development in zebra fish. This model helps to correctly identify pathogenic mutations from non- pathogenic ones.

Dr. Vinod Scaria (IGIB, New Delhi) is working on disorders caused due to mitochondrial mutations, which cover a wide range of pathologies. They have developed a software to detect heteroplasmy in mitochondria.

\section{Market concerns in Rare Disease Therapeutics}

Mr. Himanshu Bansal (Sanofi Genzyme, India) apprised the participants about the humanitarian program of Genzyme, where 650 patients are being given free enzyme replacement therapy for Gaucher's disease through the company's programme to provide therapy to the needy. In 1999, the first Indian patient received free therapy from Genzyme, although the company started its operations in India only in 2008. They now have India Charitable Access Program for a number of diseases including Gaucher and Pompe, and over 100 patients have benefited. Mr. Bansal highlighted the problems of getting sufficient number of patients for clinical trials in rare diseases, and the possibility of coordinating internationally to get enough patients. He stressed on the need to create positive ecosystem for orphan drug development in India by giving appropriate incentives, spawning collaborations with scientists and other factors including national policy which drive decision making and contribute to market policy.

\section{Way Forward}

The workshop highlighted the importance of studying "rare diseases" and pointed to large gaps in our understanding in the Indian context. A major issue is the lack of epidemiological data and adequate information about phenotype-genotype correlation in rare diseases in India. It was felt that this should be taken up on an urgent basis as the data will be required for formulating a policy on rare diseases. Development and implementation of training/ information modules on rare diseases; guidelines for diagnosis, prevention and management of rare diseases; establishment of networks of laboratories/ institutions for diagnosis, counselling and management in a referral chain mode; establishment of registries, identification of rare diseases for priority action, enactment of suitable legislation/amendment of current Drugs and Cosmetic act as per the needs of drug development/production for rare diseases, are some of the important measures suggested during the workshop. It was also felt that there is a need to have greater collaboration between clinicians and basic scientists in order to understand many aspects of the biology of these diseases that will be helpful in developing new therapeutics. Experts participating in this workshop emphasized that different science academies in India, various ministries and science departments of Government need to play complementary roles to raise awareness and improve management by accelerating research and development as well as improving the lot of this section of society so that persons afflicted with rare diseases remain healthier and useful citizens for longer periods of time. INSA and other science academies can contribute to the process by serving as potential fora to develop materials for increasing awareness, training, policy/legislation changes and creation/ strengthening of networks. 